International Journal of Engineering \& Technology, 7 (4.6) (2018) 350-353
International Journal of Engineering \& Technology
SPC
Website: $w$ ww.sciencepubco.com/index.php/IJET
Research paper

\title{
Network Efficiency Amendment Utilizing Cloud Radio Access Network In Mobile Communications
}

\author{
${ }^{1}$ Karthikala.P, ${ }^{2}$ Dr.C.Arunachala Perumal, ${ }^{3}$ Ms.M.Ramya \\ ${ }^{l}$ PG Student,${ }^{2}$ Professor, ${ }^{3}$ Assistant Professor \\ ${ }^{1,2,3}$ Department of Electronic and Communication Engineering \\ S.A. Engineering College, Thiruverkadu, Tamilnadu. \\ *Corresponding author E-mail: ${ }^{1}$ karthikala21@gmail.com
}

\begin{abstract}
Mobile data traffic is finding exponential growth currently in telecommunications industry. It has become important to concentrate on both spectral and energy efficiencies in utilizing cellular networks under green communication standpoint. Thus, for $5 \mathrm{G}$ the utmost priority is that to increase data traffic and reduce the total network energy ingesting by half. The proposed work is to design the Cloud Radio Access Network (C-RAN) with energy efficient, flexible and capacity-enhanced features by effectively bundling and establishing relation between BBU and RRU utilizing Catechistic technique. Mathematical results with realistic parameters prove that the projected optimization design clearly improve the energy efficiency of C-RAN's compared to standard schemes.
\end{abstract}

Keywords: Bundling up, Catechistic algorithm, Data sharing, Energy efficient, Mapping RRH and BBU

\section{Introduction}

It is predictable that the supplies for high speed mobile networking including high quality video streams, social networking and machine to machine communication will increase in hundreds times by 2020 . This can be due to the increase of mobile users besides the high expectation to get higher quality of services. In addition, such growth will come in parallel with the promising 5G cellular networks to handle all network traffic and provide high quality services. In order to reflect such growth, operators such as AT\&T and Telecoms will need to modify their infrastructure to handle such increase in the number of users and services expected. This can cost operators a lot in terms of capital and operating expenses which are expected to grow exponentially in such cases. On the other hand, the revenue of such operators is decreasing as they are mostly experiencing low growth in their incomes.

The division of the cellular networks that requires being adapted to handle such growth is called Radio Access Networks (RAN). RAN included base stations and users associations wirelessly to the system in addition to handling user signaling and managements. This is noticeably taking the maximum costs in such systems as base stations operation and organization get actually difficult and charge huge. Additionally, to answer user's growth and necessity, additional base stations will need to be operated in the same area so that a base station does not get congested. Though, having additional base station will bring in the difficulty of base stations synchronization so that they will not influence each other. The system will become progressively difficult in requisites of physical operation and synchronization. Furthermore, the charge of such system will be much more such that no operator will be able to handle such cost and hence a better cost efficient resolution required to be planned.

And so forth, Cloud Radio Area Networks (C-RAN) [1] was first introduced by China Mobile in 2009 to house such expansion in mobile communications. It is a new mobile structural design that has the prospective to manage as many base stations as the network. In C-RAN, the baseband and channel processing is visualized and shared among operators in a centralized baseband puddle. Such systematization and sharing allows for more lively traffic handling and improved consumption of resources including base stations stationing. Such structural design would have the potential to reduce the operating cost as base stations are visualized instead of actually stationing in all areas. Adding up, it reduces the energy and power consumption compared to conventional networks due to the reality that base stations will be deployed on the same physical device.

The main thought behind C-RAN is to split down the traditional base station into a digital utility unit, known as the Baseband Unit (BBU), and an economical radio utility unit, known as the Remote Radio Head (RRH). While RRHs are disseminated across many sites, BBUs are shared in a cloud data center. Further, RRHs are associated to BBUs through high-performance optical front haul links. RRHs only have fundamental broadcast and reception functionalities, but BBUs handle figuratively intensive baseband processing. Traditionally, each BBU is assigned to one RRH. In this setting, radio and computing resources, dimensioned for the peakload conditions, are exclusively dedicated to one RRH. This leads to unproductive resource consumption, predominantly at low-load environment [2]. On the other hand it is promising to accomplish numerical gain by clustering RRHs adequately. Naturally as a utility 
of network load environment, a one-to-many logical bundling may be realized, such that RRHs pool the resources of one single BBU, increasing network energy efficiency

\section{Related Work}

Generally, the Energy efficiency can be calculated by means of power utilization standard (watts/unit area) [3]. But commonly accepted standard is the percentage of the network throughput and the total power consumption (bits/Joule) [4], [5], [6]. To perk up the network efficiency, there are several options of increasing the data rate, or decreasing the RRH transmit power, or turning off the RRHs, bundling the RRH and BBU effectively or reducing the front haul rate for power cutback, and also by implementing any combination henceforth.

Amongst the roundabout approaches, [4] sets the user rates and convert the energy efficiency gain difficulty into a power minimization crisis. Even if data-sharing and compression methodologies are considered, the partial front haul capability constraint is not considered. The effort of [7] captures a diverse power minimization idea for downlink C-RANs under the datasharing methodology, where in users are split into several groups. Applying the arbitrary matrix assumption, [6] ideates heuristic user association (UA) methods that maximize the corresponding energy efficiency under data sharing. Conversely, the proposal of [8] openly increases the energy efficiency using the compression-based strategy. Nevertheless, in [8] the data-sharing condition and the power consumption at the front haul links are not addressed. It is significantly clear that the abovementioned proposals focus on single-hop transmission networks only, while a realistic BBU is normally connected to RRHs via a number of routers over a multihop front haul network [9], [10]. Recent works in [9] try to increase the network throughput of a downlink multi-hop C-RAN, where network programming is considered important to utilize the front haul associations in the multi-hop transmission. Conversely, the maximization of energy efficiency has not yet been achieved.

Furthermore, RRH grouping has been projected, in the journalism, for many reasons. In [11], the authors propose a lively clustering to introduce inter-cell cooperation. RRHs that are mapped to one BBU concurrently broadcast the similar signal. This makes the most of the system effectiveness. The authors in [12], bring in clustering to capitalize on user fulfillment. Signaling consignment is bargained, without negotiation on user quality of service. They also improve network energy utility, uttered through the number of dynamic BBUs. In [13], the proposal is on a active channel allotment. They actively transform the BBU-RRH relation, when non-interfering channels are allotted to adjacent RRHs. The projected resolution has a straight impact on network's energy efficiency. But, we consider that efficiency could be even more improvised using other optimal algorithms and the network utilities better used.

This paper concentrates on the C-RAN downlink with an ability of multi-hop and capacity-limited front haul transmission. The intention is to directly maximize the network energy efficiency by BBU-RRH bundling using Catechistic technique, in order to enhance radio resource utilization and power consumption. We not only depend over the number of dynamic BBUs, but also assume a power minimization replica to closely examine the impact of bundling up the RRH on network efficiency.

The rest of the paper is structured as follows: Section 3 describes the system model under consideration. Section 4 introduces our Catechistic algorithm, while section 5 put together and explain the optimization solution for the data-sharing strategy. Performance evaluation is presented in section 6 . Section 7 concludes the paper.

\section{System Model}

We consider a Cloud Radio Access Network topology which comprised of Primary Users (PU), Secondary Users (SU), Radio Resource Head (RRH) and Primary Base Station (PBS) /central base band unit (BBU). Fig. 1 and Fig 2 demonstrate the downlink of a common C-RAN representation with multi-hop and single-hop links, where the baseband unit (BBU) in the hub network connects to a set of $\mathrm{m}$ RRHs $r r \square_{1}, \ldots, r r \square_{m}$, via a front haul network of $R$ routers and $N$ noiseless front haul links [4], [9], [14], The objects are the RRHs with different average demands $\delta_{1}, \ldots . \delta_{m}$ and the BBUs are the containers with a highest Capacity limit, $\varepsilon$. Focus to the BBU maximum capacity and to the environmental adjacency conditions, the BBU-RRH relation must be established in such a way to decrease the number of lively BBUs, while affording the equal level of Quality of Service as that of a one-to-one relationship. There exists a neighboring relation, given by $m_{i j}=1$, between $m_{i}$ and $m_{j}$ when they are geographically nearby. While they have no sharing border, $m_{i j}=0$.

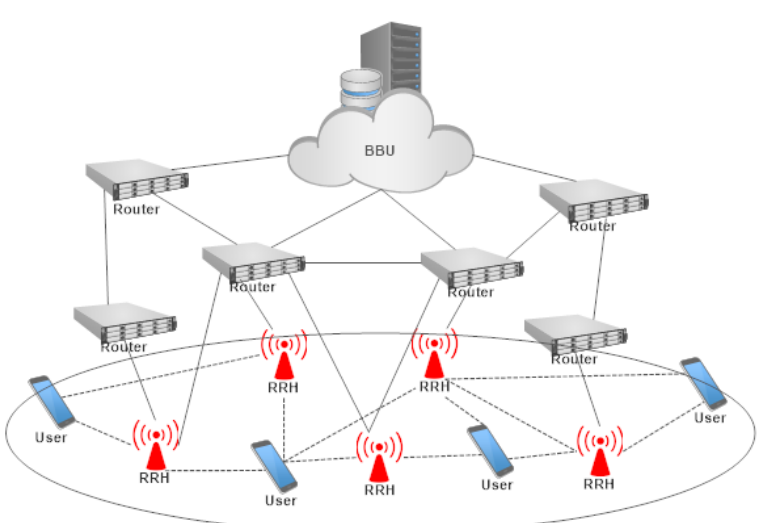

Fig. 1: A general C-RAN structure with multi-hop links

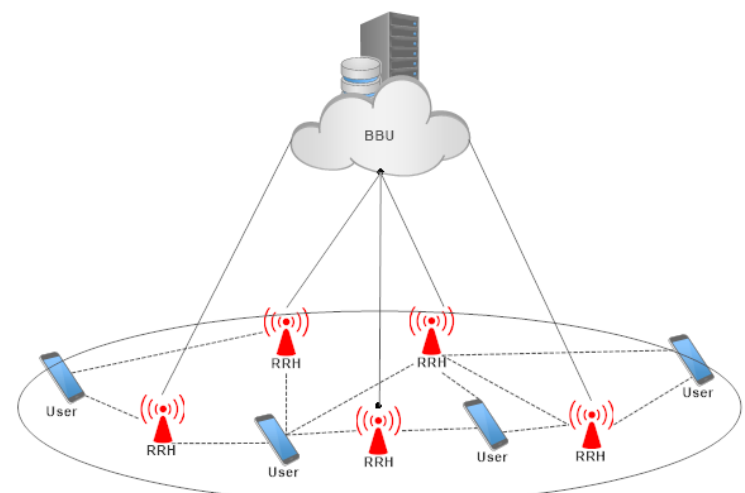

Fig. 2: A general C-RAN structure with single-hop links

\section{Catechistic Algorithm}

Inspired by the Agglomerative Hierarchical Clustering (HAC) algorithm, we propose an algorithm that attempts to fill up all vacant, such that channel bandwidth is utilized to the fullest. The individuality of our algorithm, in comparison with the HAC strategy, is that it takes into concern the physical adjacency restriction. Algorithm 1 describes our catechistic method. We indicate by $\mathrm{m}$ set of RRHs, $\delta$ their load/demand, ' $\Upsilon$ set of resource blocks in RRH and $\omega$ be the cluster set. 
Algorithm follows the below steps

1. Get the demands $\delta$ of all RRHs

2. Select the RRH with largest demand $\delta$ (max).

3. Collect the neighbors of high demand RRH based on the distance $\varepsilon$ and their corresponding loads $\delta$.

4. Among the neighbors, find the RRH with least demand $\delta($ least $)$

5. Check whether the sum of high demand and least demand not exceeds capacity of BBH,

6. Repeat the bundling till the above condition is over ridden.

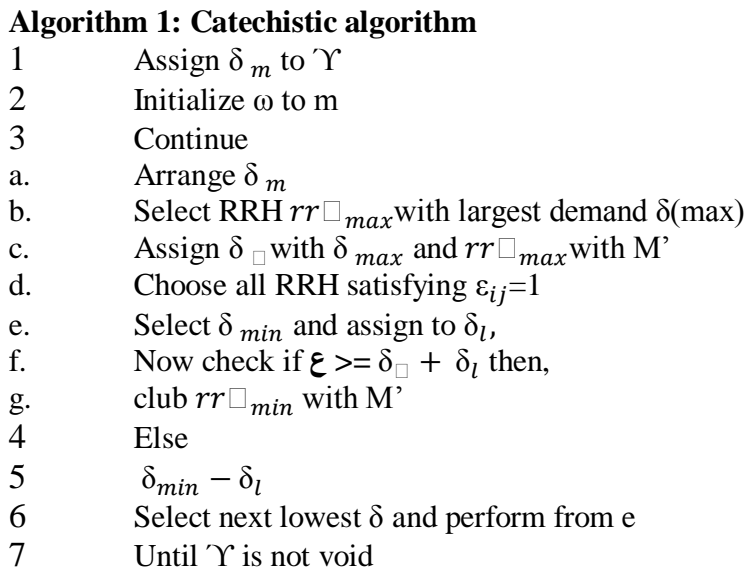

\section{Optimization Solution for the Data-Sharing Strategy}

With the data-sharing methodology, users' anticipated signals are transmitted by the BBU to a selected set of RRHs which then considerately execute pre-coding and transmit the coded signals to the users [4], [9], [14]. Below are the mentioned phases of the established communication.

\section{- Data broadcast with Network programming at BBU}

For data transmission, every router will copy and transmit onward its received data to the adjacent routers, or use a type of coding to create encoding and decoding messages for the information transmission [15]. As given in [16], the copy-and-forward method is not the best while the coding operation at routers achieves highest capacity. In [9], the proposal utilize a network coding strategy that consists of a routing plan and a code transfer to decide the rate and substance of each flow of data being transmitted across the network. The proposal of [17] shows that interference coding only offer minimal output gain over self-determining coding. Hence, this paper presumes that multicast transmission is routed and coding is done independently.

- $\quad$ Signal programming and broadcast by RRHs

In receipt of the message from all sessions via the multi-hop downlink network, an RRH generates a baseband signal. The RRH is subjected to the average transmit power. Flat-fading channel from RRH remains unaffected during the transmission and are accessible at the BBU and RRHs [9] [18].

\section{- User relationship and RRH stimulation at Radio}

\section{Access links}

An RRH is always dedicated to serve a user if and only if the corresponding coder of the message symbol is not a zero matrix and assures that no user is allotted to a dormant RRH.
We try to increase the network energy efficiency which is given as the ratio of the achievable sum rate/ network throughput and the total power utilization. The optimization solution for the multi-hop C-

RAN with the data-sharing method is planned as follows.

$\mathrm{P}_{\text {optimal }} \triangleq \frac{\mathrm{R}_{\text {sum }}}{\mathrm{P}_{\text {total }}}$

\section{Performance Evaluation}

From design perspective, we utilize a network topology made of 10 primary users, 25 secondary users and 1 primary base band unit. Every RRH is imitated with 2 antennas and each user with 1 antenna for communication. The snooze mode and the lively mode of each RRH utilize 56W and 84W of power, respectively. NS3 Simulations are performed for various load/demand conditions, like low, medium, and high load environment. The LTE parameters and the adopted parameters used in our numerical examples are listed in table I. 16-QAM modulation scheme is assumed to be utilized. Performance results are averaged and shown with $95 \%$ target.

Table I:

\begin{tabular}{|l|l|}
\hline PARAMETERS & VALUES \\
\hline Coverage Distance between RRHs & $5 \mathrm{~km}$ \\
\hline Total Bandwidth & $20 \mathrm{MHz}$ \\
\hline Max RRH transmit power & $24 \mathrm{dBm}$ \\
\hline Data Rate & $100 \mathrm{Mbps}$ \\
\hline Inter packet interval & $100 \mathrm{~ms}$ \\
\hline Resource block duration & $0.5 \mathrm{~ms}$ \\
\hline
\end{tabular}

The operational or network efficiency of radio resources are defined as a function of average RRH load/demands. It is proposed as the ratio of the entire number of used resource blocks in all RRHs to the number of operating BBUs.

From graphical results Fig. 3 and Fig 4, we infer that at low demand criteria, the operational efficiency of resources is achieved to be almost $80 \%$ with bundling RRH.

And at intermediate demand, the operational performance has similar numerical as for low demand conditions with bundling RRH. It illustrates that the projected algorithm performs much better than the standard methods in terms of the energy efficiency. To conclude, the bundling of RRH and establishing proper data sharing strategy has a huge impact on the number of operating BBUs, henceforth reducing network power consumption without compromising user Quality of Service (QoS).

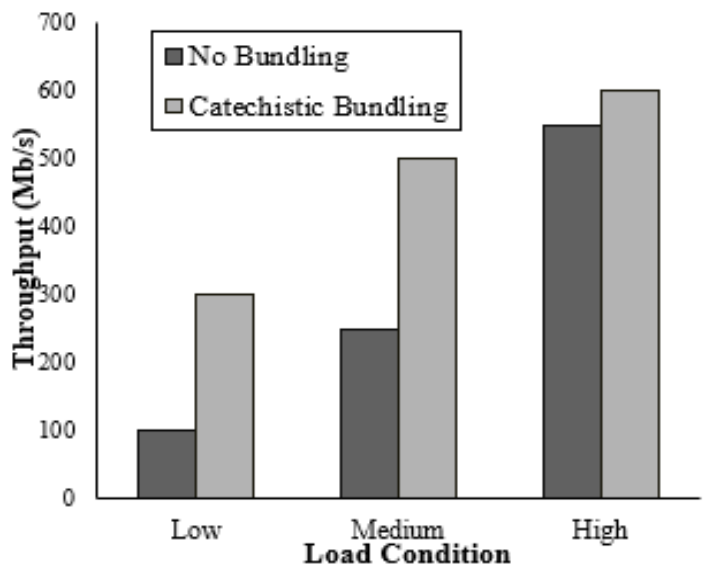

Fig. 3: Various Load Conditions Throughput for non bundling and Catechistic Approach 


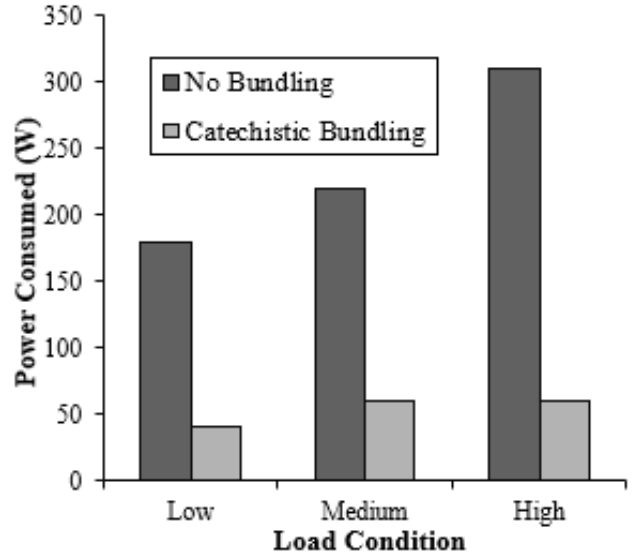

Fig. 4: Various Load Conditions Power Consumed for non bundling and Catechistic Approach

\section{Conclusion}

In this paper, we have addressed the RRH bundling using catechistic strategy in Cloud Radio Access Network and presented optimal solutions. Our effort initiated important advancement on energy efficiency and operational efficiency, at low and intermediate demand conditions, without concession on user quality. The planned proposal offers optimal performance optimal solution, at low and medium load conditions, with reduced computational difficulty. As future work, we propose to incorporate the inter-cell interferences and handover issues and study their impact on the RRH bundling.

\section{References}

[1] Online:https://www.cse.wustl.edu/ jain/cse574-16/ftp/cloudran/

[2] Hussein Taleb, Melhem El Helou, Kinda Khawam, Samer Lahoud, and Steven Martin, "Centralized and Distributed RRH Clustering in Cloud Radio Access Networks", Jul 2017

[3] F. Richter, A. J. Fehske, and G. P. Fettweis, "Energy efficiency aspects of base station deployment strategies for cellular networks," in Proc. Int. IEEE Veh. Technol. Conf. (VTC), Sep. 2009, pp. 1-5.

[4] B. Dai and W. Yu, "Energy efficiency of downlink transmission strategies for cloud radio access networks," IEEE J. Sel. Area. Commun., vol. 34, no. 4, pp. 1037-1050, Apr. 2016.

[5] P. Gandotra, R. K. Jha, and S. Jain, "Green communication in next generation cellular networks: A survey," IEEE Access, vol. 5, pp. 11 727- $11758,2017$.

[6] J. Zuo, J. Zhang, C. Yuen, W. Jiang, and W. Luo, "Energy efficient user association for cloud radio access networks," IEEE Access, vol. 4, pp.2429-2438, 2016.

[7] R. Sun, M. Hong, and Z. Q. Luo, "Joint downlink base station association and power control for max-min fairness: Computation and complexity," IEEE J. Sel. Areas Commun., vol. 33, no. 6, pp. 10401054, Jun. 2015.

[8] K. G. Nguyen, Q. D. Vu, M. Juntti, and L. N. Tran, "Energy efficient preceding C-RAN downlink with compression at fronthaul," in Proc.IEEE Int. Conf. Commun. (ICC), May 2017, pp. 1-6.

[9] L. Liu andW. Yu, "Cross-layer design for downlink multihop cloud radio access networks with network coding," IEEE Trans. Signal Process., vol. 65, no. 7, pp. 1728-1740, Apr. 2017.

[10] S. H. Park, O. Simeone, O. Sahin, and S. Shamai, "Multihop backhaul compression for the uplink of cloud radio access networks," IEEE Trans.Veh. Technol., vol. 65, no. 5, pp. 3185-3199, May 2016.

[11] Y. Du and G. de Veciana, "Wireless Networks without Edges: Dynamic Radio Resource Clustering and User Scheduling," in Proc. IEEE Conference on Computer Communications (INFOCOM), April 2014.

[12] K. Sundaresan, M. Y. Arslan, S. Singh, S. Rangarajan, and S. V. Krishnamurthy, "FluidNet: A Flexible Cloud-based Radio Access
Network for Small Cells," in Proc. ACM International Conference on Mobile Computing \& Networking, September 2013.

[13] K. Wang, M. Zhao, and W. Zhou, "Traffic-aware Graph-based Dynamic Frequency Reuse for Heterogeneous Cloud-RAN," in Proc. IEEE Global Communications Conference (GLOBECOM), December 2014.

[14] M. Tao, E. Chen, H. Zhou, and W. Yu, "Content-centric sparse multicast beamforming for cache-enabled cloud RAN," IEEE Trans. Wireless Commun., vol. 15, no. 9, pp. 6118-6131, Sep. 2016.

[15] J. Yuan, Z. Li, W. Yu, and B. Li, "A cross-layer optimization framework for multihop multicast in wireless mesh networks," IEEE J. Sel. Areas Commun., vol. 24, no. 11, pp. 2092-2103, Nov. 2006.

[16] R. Ahlswede, N. Cai, S. Y. R. Li, and R. W. Yeung, "Network information flow," IEEE Trans. Inf. Theory, vol. 46, no. 4, pp. 1204 1216, Jul. 2000

[17] Z. Li, B. Li, D. Jiang, and L. C. Lau, "On achieving optimal throughput with network coding," in Proc. IEEE INFOCOM, vol. 3, Mar. 2005, pp.2184-2194 vol. 3.

[18] S. H. Park, O. Simeone, and S. S. Shitz, "Joint optimization of cloud and edge processing for fog radio access networks," IEEE Trans. Wireless Commun., vol. 15, no. 11, pp. 7621-7632, Nov. 2016.

[19] Hussein Taleb, Melhem El Helou, Samer Lahoud, Kinda Khawam, and Steven Martin, "An Efficient Heuristic for Joint User Association and RRH Clustering in Cloud Radio Access Networks", Jun 2018

[20] Tung T. Vu, Duy T. Ngo, Minh N. Dao, Salman Durrani, Duy H. N. Nguyen and Richard H. Middleton, Energy Efficiency Maximization for Downlink Cloud Radio Access Networks with Data Sharing and Data Compression, May 2018

[21] Yawen Chen,Xiangming Wen, Zhaoming Lu, Hua Shao, "Energy Efficient Clustering and Beamforming for Cloud Radio Access Networks", Sep 2016

[22] Lei Zhang, Atta ul Quddus, Efstathios Katranaras, Dirk Wubben, Yinan Qi, Rahim Tafazolli, "Performance Analysis and Optimal Cooperative Cluster Size for Randomly Distributed Small Cells under Cloud RAN", Apr 2016

[23] Binbin Dai, and Wei Yu, "Energy Efficiency of Downlink Transmission Strategies for Cloud Radio Access Networks", Apr. 2016

[24] Shun-Cheng Zhan, Dusit Niyato, "A Coalition Formation Game for Remote Radio Heads Cooperation in Cloud Radio Access Network", Apr 2016

[25] K. Boulos, M. E. Helou, and S. Lahoud, "RRH Clustering in Cloud Radio Access Networks," in Applied Research in Computer Science and Engineering (ICAR), Oct 2015. 